\title{
Change of Hypermucoviscosity in the Development of Tigecycline Resistance in Hypervirulent Klebsiella pneumoniae Sequence Type 23 Strains
}

\author{
Suyeon Park ${ }^{\dagger}$, Haejeong Lee ${ }^{\dagger}(\mathbb{D}$, Dongwoo Shin and Kwan Soo Ko*(1) \\ Department of Microbiology and Samsung Medical Center, Sungkyunkwan University School of Medicine, \\ Suwon 16419, Korea; parks024@naver.com (S.P.); hjlee7834@skku.edu (H.L.); shind@skku.edu (D.S.) \\ * Correspondence: ksko@skku.edu; Tel.: +82-31-299-6223 \\ + These authors are equally contributed to this work.
}

Received: 11 September 2020; Accepted: 7 October 2020; Published: 10 October 2020

\begin{abstract}
In this study, we developed tigecycline resistance in Klebsiella pneumoniae ST23 strains in vitro and investigated the change in virulence associated with hypermucoviscosity. In vitro-induced tigecycline-resistant (TGC-IR) K. pneumoniae mutants were obtained from three tigecycline-susceptible (TGC-S) strains, belonging to ST23 and serotype K1, by culturing in media with tigecycline in a stepwise manner. An antimicrobial susceptibility test, string test, mucoviscosity assay, and capsular polysaccharide (CPS) quantification were performed. Biofilm formation and serum resistance were evaluated, and survival rates of bacterial strains in fruit flies and macrophages were measured. Alterations of $r p s J, r a m R, \operatorname{soxR}, a c r R$, and $\operatorname{mar} R$ genes were investigated and the expression levels of $\operatorname{ram} A$ and efflux pump genes were evaluated. The hypermucoviscosity phenotype was dramatically decreased in the TGC-IR mutants. Reduced CPS production in TGC-IR mutants was also identified. Increased resistance to most other antimicrobial agents was found in TGC-IR mutants. In addition, the TGC-IR mutants exhibited reduced biofilm formation, low serum resistance, and decreased survival rates within fruit flies and macrophages. Our study shows that development of tigecycline resistance in hypervirulent K. pneumoniae strains result in defects in virulence associated with hypermucoviscosity.
\end{abstract}

Keywords: tigecycline resistance; hypermucoviscosity; hypervirulent Klebsiella pneumoniae; ST23

\section{Introduction}

Klebsiella pneumoniae is a Gram-negative bacillus that is responsible for both community-acquired and nosocomial infections including pneumonia, urinary tract infections, bacteremia, and liver abscesses [1]. Since the mid-1980s, hypervirulent K. pneumoniae (hvKP), generally associated with the hypermucoviscosity (HV) phenotype, has emerged as a clinically significant pathogen responsible for serious disseminated infections [2,3]. The HV phenotype of hvKP is typically due to the increased production of capsular polysaccharide (CPS) and the presence of specific virulence genes $[4,5]$. Notably, sequence type 23 (ST23) is the most commonly described among hypermucoviscous K. pneumoniae isolates and is strongly correlated with capsular serotype $\mathrm{K} 1$ and liver abscess $[1,6,7]$.

An increase in carbapenem resistance in K. pneumoniae worldwide has limited the available treatment options for the bacterium. Tigecycline is a last-resort antibiotic reserved for treatment in carbapenem-resistant K. pneumoniae infection [8]. Thus, tigecycline resistance means that options for the treatment of carbapenem-resistant K. pneumoniae infections are virtually eliminated. Tigecycline inhibits protein translation by binding to the $30 \mathrm{~S}$ subunit of the bacterial ribosome, which ultimately prevents bacterial growth [9]. However, reports of tigecycline resistance have increased year by year [10]. In K. pneumoniae, resistance to tigecycline is mainly attributed to overproduction of efflux 
pumps (AcrAB, OqxA, KpgAB) [11] and to mutations in efflux pump regulator genes $(\operatorname{ram} A$, soxR, $\operatorname{mar} R, \operatorname{acr} R$ ) [12]. Mutations in $\operatorname{ram} R$, which encodes a transcriptional repressor belonging to the TetR family, are also responsible for tigecycline resistance by leading to the overexpression of $\operatorname{ram} A$. Moreover, mutations in rpsJ, the gene that encodes the ribosomal S10 protein, could confer reduced susceptibility to tigecycline in K. pneumoniae [13].

In this study, we investigated the association between tigecycline resistance and $\mathrm{HV}$ in hvKP isolates belonging to ST23. We described the decreased $\mathrm{HV}$ in three in vitro tigecycline-induced-resistant (TGC-IR) mutants of serotype K1 K. pneumoniae ST23 strains. We compared the phenotype, virulence, and CPS production in tigecycline-susceptible (TGC-S) and TGC-IR isolates. In addition, we performed qRT-PCR and sequencing of the genes related to tigecycline resistance in K. pneumoniae.

\section{Materials and Methods}

\subsection{Bacterial Strains and In Vitro Induction of Tigecycline-Resistant Mutants}

Three TGC-S K. pneumoniae strains, SMC1204-109, SMC1207-200, and SMC1208-086, were used in this study. They were obtained from patient blood samples at Samsung Medical Center (Seoul, Korea). The strains belonged to ST23 in multilocus sequence typing analysis. For TGC-S strains, MLST and K serotyping were performed as described previously [14,15]. The TGC-S strains were experimentally evolved to TGC-IR mutants using a previously described method [16]. Briefly, the three TGC-S K. pneumoniae strains were grown overnight at $37^{\circ} \mathrm{C}$ and subcultured in Luria-Bertani (LB) broth with a serially increasing concentration of tigecycline ( 0.5 to $64 \mathrm{mg} / \mathrm{L}$ ). The successfully developed TGC-IR mutants, SMC1204-109-IR, SMC1207-200-IR, and SMC1208-086-IR, were used in this study.

\subsection{Antimicrobial Susceptibility Testing}

Antimicrobial susceptibility testing was performed using the broth microdilution method following Clinical and Laboratory Standards Institute (CLSI) guidelines [17]. The minimum inhibitory concentrations (MICs) of ten antimicrobial agents including tigecycline, tetracycline, ampicillin, gentamicin, cefotaxime, ceftazidime, meropenem, amikacin, ciprofloxacin, piperacillin-tazobactam, and colistin were determined. The MICs of tigecycline in the presence of efflux pump inhibitors, carbonyl cyanide 3 -chlorophenylhydrazone (CCCP), and phenyl-arginine- $\beta$-naphthylamide (PA $\beta N$ ) were also measured. Antimicrobial susceptibility was defined according to the CLSI breakpoints [17], with Escherichia coli ATCC 25922 and Pseudomonas aeruginosa ATCC 27853 as reference strains. Tigecycline MICs were interpreted according to EUCAST guidelines [18]. Tigecycline susceptibility of bacteria was defined using only Escherichia coli ATCC 25922 as the reference strain. All tests were performed in duplicate with three biological replicates per strain.

\subsection{String Test}

The HV phenotype of the TGC-S and TGC-IR strains was evaluated using the string test as described previously [1]. All tested isolates were cultured overnight on blood agar plates at $37^{\circ} \mathrm{C}$, and bacterial colonies were then stretched with an inoculation loop.

\subsection{Mucoviscosity Assay and CPS Quantification}

Because the supernatant of HV strains remains turbid after centrifugation, measurement of the turbidity after centrifugation can serve as a quantitative indicator of HV [19]. Therefore, the mucoviscosity assay was performed following a previously described methodology [20]. Bacterial strains were grown in LB broth at $37^{\circ} \mathrm{C}$ overnight with shaking. The samples were centrifuged at low speed $(1000 \times g)$ for $5 \mathrm{~min}$ and the absorbance of supernatants was measured at $600 \mathrm{~nm}\left(\mathrm{OD}_{600}\right)$.

To quantify CPS in TGC-S and TGC-IR K. pneumoniae strains, we extracted and measured the amount of CPS from bacteria as described previously [16]. Bacterial cultures were mixed with $1 \%$ Zwittergent 3-14 detergent (Sigma-Aldrich, St. Louis, MO, USA) in $100 \mathrm{mM}$ citric acid and then, 
the mixtures were incubated at $50{ }^{\circ} \mathrm{C}$ for $30 \mathrm{~min}$. After centrifugation, supernatants were transferred into new tubes, and $1 \mathrm{~mL}$ of absolute ethanol was added. The pellets were dissolved in $100 \mu \mathrm{L}$ of distilled water, and then, $600 \mu \mathrm{L}$ of $12.5 \mathrm{mM}$ boric acid in $\mathrm{H}_{2} \mathrm{SO}_{4}$ was added to each tube. The mixtures were vigorously vortexed, incubated at $100{ }^{\circ} \mathrm{C}$ for $5 \mathrm{~min}$, and then, cooled. Next, $10 \mu \mathrm{L}$ of 3-hydroxydiphenol (Sigma-Aldrich, St. Louis, MO, USA) was added to the mixture and the absorbance was measured at $520 \mathrm{~nm}$. The glucuronic acid concentration in each sample was determined from a standard curve of glucuronic acid and expressed as micrograms per $10^{9} \mathrm{CFU} / \mathrm{mL}$.

\subsection{Biofilm Formation and Serum Resistance Assays}

To evaluate biofilm formation, 96-well microtiter plate assays were performed with crystal violet as described previously [21], with minor modifications. Briefly, the mid-log phase bacterial cultures were added to a flat bottom plate and incubated at $37^{\circ} \mathrm{C}$. After $24 \mathrm{~h}$, all of the bacterial cultures and LB broth (used as the negative control) were removed and washed twice with phosphate-buffered saline (PBS). Then, $0.1 \%$ crystal violet solution was added to the plate for staining before incubation for $20 \mathrm{~min}$ at room temperature. The solution was completely removed and washed three times with PBS. The walls were dried, and bound dye was solubilized with $200 \mu \mathrm{L}$ of $95 \%$ ethanol and quantified by an xMark Microplate Absorbance Spectrophotometer (Bio-Rad, Hercules, CA, USA) at 540 nm. Each assay was performed in duplicate and repeated three times independently.

The serum resistance assay was performed as described previously [22]. Normal human serum diluted to $20 \%$ (NHS, Innovative Research, Novi, MI, USA) was treated to the mid-log phase bacterial cultures. As a control, heat-inactivated human serum (HIS) was used to determine the bactericidal effect of NHS. After $3 \mathrm{~h}$ of incubation with shaking, the mixtures were serially diluted and plated on blood agar. The number of colony-forming units (CFUs) that survived after treatment with NHS was compared with the number of CFUs that survived after treatment with HIS. All assays were performed three times and the results are represented as survival percentage.

\subsection{Drosophila Melanogaster (Fruit Fly) Infection}

To assess the virulence of TGC-S and TGC-IR strains, a fruit fly infection model was used with the thoracic needle-prick method as described previously [22]. Three- to five-day-old female flies were infected with bacterial cultures $\left(\mathrm{OD}_{600}\right.$ of 20) using an ultra-fine needle (BD Biosciences, San Jose, CA, USA). A pure PBS injection was used as a negative control. Twenty flies were infected for each bacterial strain; the mortality of flies was monitored until $72 \mathrm{~h}$ post infection. The experiments were performed three times independently.

For quantification of viable bacteria, infected flies from each bacterial strain group were individually immersed in $100 \mu \mathrm{L}$ of PBS. Five flies from each strain were homogenized by a Teflon pestle and serially diluted. Then, the mixtures were plated onto LB agar containing $100 \mathrm{mg} / \mathrm{L}$ of ampicillin. The number of CFUs per fly was counted.

\subsection{Macrophage Infection Assay}

A macrophage infection assay was performed to assess the survival rate of bacteria using a previously described method [23], with slight modifications. Macrophage-like line J774A.1 cells were grown in Dulbecco's Modified Eagle Medium (DMEM) (Welgene, Gyeongsan, Korea) supplemented with 10\% fetal bovine serum (FBS) (Gibco) and 1\% antibiotic-antimycotic solution (Thermo, Waltham, MA, USA). A monolayer of macrophage cells was prepared in a 12-well tissue culture plate before bacterial infection. Macrophage cells $\left(1 \times 10^{6}\right)$ were washed with Dulbecco's phosphate-buffered saline (DPBS) (Welgene, Gyeongsan, Korea) and incubated in DMEM with FBS for $1 \mathrm{~h}$. Bacterial isolates cultured overnight were inoculated at ratio of 20 bacteria per macrophage (MOI 20). The cells were incubated for $30 \mathrm{~min}$ at $37^{\circ} \mathrm{C}$ to permit phagocytosis and the supernatants were washed with DPBS three times. After infection with bacterial strains, pre-warmed DMEM supplemented with a high concentration of gentamicin $(150 \mathrm{mg} / \mathrm{L})$ was added to kill extracellular bacteria and incubated for $1 \mathrm{~h}$. 
The wells for the 0 -h time point were washed with DPBS and treated with $1 \%$ Triton X-100 immediately. For the 4- and 20-h time point samples, the washed cells were supplemented with a low concentration of gentamicin (15 mg/L) and treated with Triton X-100 at each time point. Then, the diluted mixture from each well was spread on blood agar plates for the calculation of CFUs. The survival percentages of bacteria after 4 and $20 \mathrm{~h}$ were calculated compared to the CFUs at the $0 \mathrm{~h}$ time point. All experiments were performed in duplicate and repeated three times independently.

\subsection{Sequencing for Assessing Gene Mutations}

For analysis of nucleotide alterations in $\operatorname{ramR}, \operatorname{marR}, \operatorname{acrR}, \operatorname{sox} R$, and $r p s$ J, we sequenced the genes with primers described previously [12,24]. Genomic DNA was extracted from the K. pneumoniae strains using a gDNA extraction kit (iNtRON, Seongnam-si, Korea) according to the manufacturer's instructions. Sequencing of the PCR products was conducted by Macrogen (Seoul, Korea).

\subsection{Quantitative RT-PCR}

To compare the alteration of gene expression according to tigecycline resistance in K. pneumoniae, quantitative real-time PCR (qRT-PCR) was performed [25]. Total RNA was extracted from mid-log phase bacterial cultures $\left(\mathrm{OD}_{600}\right.$ approximately 0.5) using the Qiagen RNeasy Mini kit (Qiagen, Hilden, Germany) according to the manufacturer's instructions. After removal of contaminating DNA from the RNA samples, reverse transcription reactions were performed using the reverse transcription premix kit (iNtRON, Seongnam-si, Korea). To quantify the target genes, qRT-PCR was performed using TB Green Premix Ex Taq (TaKaRa, Shiga, Japan) on the QuantStudio 6 Flex Real-Time PCR system (Applied Biosystems, CA, USA) with the primers listed in Table S1. The fold-changes were calculated according to the comparative threshold cycle $\left(\Delta \Delta \mathrm{C}_{\mathrm{T}}\right)$ method, using the rpoB gene as the reference. The experiments were repeated with three independent cultures and each sample was tested in duplicate.

\subsection{Statistical Analyses}

Statistical analyses were performed using Prism version 3.00 for Windows (GraphPad Software, San Diego, CA, USA). The differences were assessed using Student's $t$-test, one-way ANOVA with Tukey's multiple comparisons test, and a nonparametric Kruskal-Wallis test followed by Dunnett's multiple comparison test. A $p$-value of less than 0.05 was considered statistically significant ${ }^{*}, p<0.05$; $\left.{ }^{* *}, p<0.001 ;{ }^{* * *}, p<0.0001\right)$.

\section{Results}

\subsection{Decreased Hypermucoviscosity in TGC-IR Mutants}

All three TGC-S K. pneumoniae strains used in this study, SMC1204-109, SMC1207-200, and SMC1208-086, were identified as serotype K1. TGC-IR mutants were developed from TGC-S strains by culturing in media including tigecycline in a stepwise manner, showing tigecycline MICs of $>64 \mathrm{mg} / \mathrm{L}$ (Table 1).

The HV phenotype was observed in the three TGC-S K. pneumoniae strains in the string test, ranging from 25 to $35 \mathrm{~mm}$ (Figure 1). The length of the bacterial string was reduced remarkably in the TGC-IR mutants, to $8 \mathrm{~mm}$ in SMC1204-109-IR, $5 \mathrm{~mm}$ in SMC1207-200-IR, and $4 \mathrm{~mm}$ in SMC1208-086-IR (Figure 1). Such lengths were $11.4 \%$ to $32.0 \%$ of those in their parental strains.

The mucoviscosity was also investigated by measuring the absorbance of supernatants after low-speed centrifugation (Figure 2A). Consistent with the results of the string test, all three TGC-IR mutants exhibited significantly reduced turbidity, indicating low mucoviscosity, compared with that of their parental TGC-S K. pneumoniae strains. In addition, the quantity of CPS was also significantly lower (approximately 10- to 54-fold) in the TGC-IR mutants than in the TGC-S strains (Figure 2B). 
Table 1. Tigecycline minimum inhibitory concentrations (MICs) and the results of the string test in Klebsiella pneumoniae strains used in this study.

\begin{tabular}{cccc}
\hline Strains & Strain No. & Tigecycline MIC $^{\text {a }}$ & String Test \\
\hline \multirow{3}{*}{ TGC-S strains } & SMC1204-109 & $1(\mathrm{~S})$ & $25 \mathrm{~mm}$ \\
& SMC1207-200 & $0.5(\mathrm{~S})$ & $27 \mathrm{~mm}$ \\
& SMC1208-086 & $2(\mathrm{~S})$ & $35 \mathrm{~mm}$ \\
\multirow{3}{*}{ TGC-IR mutants } & SMC1204-109-IR & $>64(\mathrm{R})$ & $8 \mathrm{~mm}$ \\
& SMC1207-200-IR & $>64(\mathrm{R})$ & $5 \mathrm{~mm}$ \\
& SMC1208-086-IR & $>64(\mathrm{R})$ & $4 \mathrm{~mm}$ \\
\hline
\end{tabular}

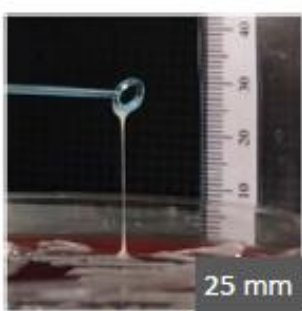

SMC1204-109

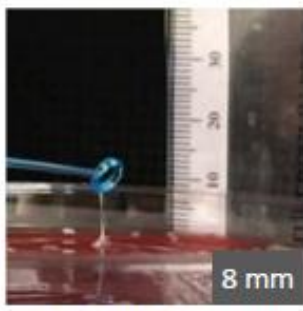

SMC1204-109-IR

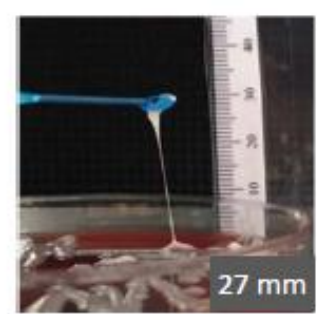

SMC1207-200

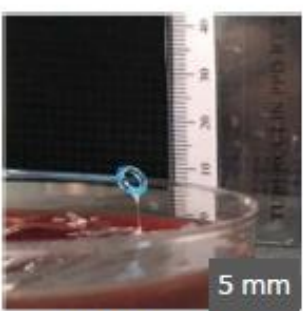

SMC1207-200-IR

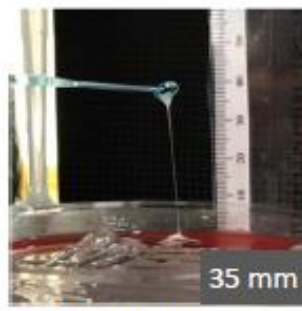

SMC1208-086

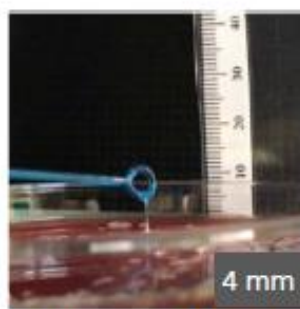

SMC1208-086-IR

Figure 1. Results of string tests. Comparison of stretched colonies between TGC-S and TGC-IR K. pneumoniae strains. Generally, a string $5 \mathrm{~mm}$ or longer was defined as positive.

A

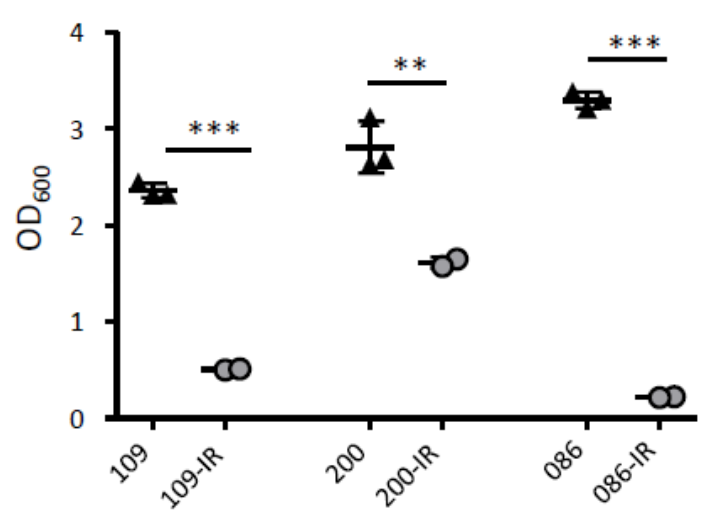

B

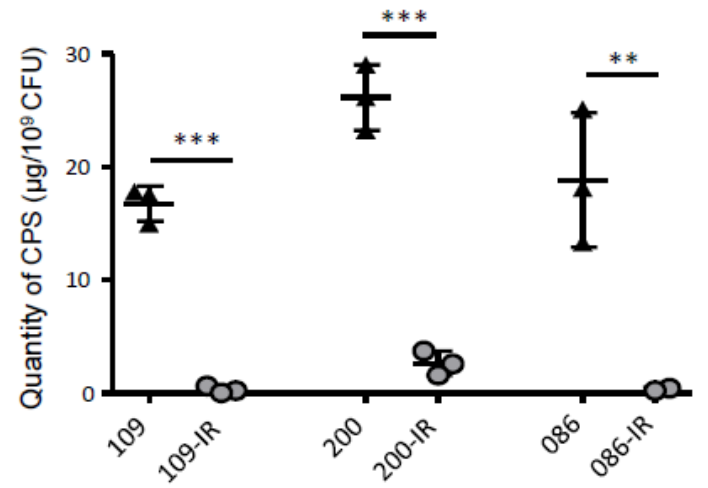

Figure 2. Results of mucoviscosity assay and capsular polysaccharide (CPS) production. (A) Mucoviscosity of TGC-S and TGC-IR isolates was measured. The absorbance of supernatants was compared after low-speed centrifugation. (B) Production of CPS was quantified in the TGC-S isolates and TGC-IR mutants. CPS biosynthesis was determined by phenol-sulfuric acid assays. ${ }^{* *}, p<0.001$; ${ }^{* * *}, p<0.0001$. 


\subsection{Antimicrobial Susceptibility Profiles}

The three TGC-S K. pneumoniae strains were susceptible to most antimicrobial agents except ampicillin (Table 2). All TGC-IR mutants showed increased MICs for tetracycline, ampicillin, cefotaxime, and piperacillin-tazobactam. While the MICs of ceftazidime, meropenem, ciprofloxacin, and colistin also increased in two TGC-IR mutants, SMC1204-109-IR and SMC1207-200-IR, it did not in the other strain, SMC1208-086-IR. The MIC of amikacin in SMC1208-086-IR was reduced compared with that of its parental strain, and gentamicin MICs were not changed in all TGC-IR mutants.

The MICs for tigecycline with efflux pump inhibitors, CCCP and PA $\beta \mathrm{N}$, were also measured. For the parental TGC-S K. pneumoniae strains, the tigecycline MIC was reduced only in SMC1204-109 with CCCP and in SMC1208-086 with PA $\beta N$ (Table 2). Their induced-resistance mutants maintained very high tigecycline MICs, $64 \mathrm{mg} / \mathrm{L}$ or higher, when $50 \mu \mathrm{M}$ of CCCP or $25 \mathrm{mg} / \mathrm{mL}$ of PAßN was added to the media. 
Table 2. Antimicrobial susceptibility profiles of Klebsiella pneumoniae strains used in this study.

\begin{tabular}{|c|c|c|c|c|c|c|c|c|c|c|c|c|c|}
\hline \multirow{2}{*}{ Strain } & \multicolumn{13}{|c|}{ MIC (mg/L) (Antimicrobial Susceptibility Category) ${ }^{a, b}$} \\
\hline & TGC & $\mathrm{TGC}+\mathrm{CCCP}^{\mathrm{c}}$ & $\mathrm{TGC}+\mathrm{Pa} \beta \mathrm{N}^{\mathrm{d}}$ & TET & AMP & GEN & CTX & CAZ & MRP & AMK & CIP & $\mathrm{P} / \mathrm{T}$ & CL \\
\hline & \multicolumn{13}{|c|}{ TGC-S strains } \\
\hline SMC1204-109 & $1(\mathrm{~S})$ & $0.5(\mathrm{~S})$ & $0.5(\mathrm{~S})$ & $2(\mathrm{~S})$ & $64(\mathrm{R})$ & $128(\mathrm{R})$ & $0.125(S)$ & $1(\mathrm{~S})$ & $0.06(\mathrm{~S})$ & $1(\mathrm{~S})$ & $0.06(\mathrm{~S})$ & $4 / 4(S)$ & $0.25(\mathrm{~S})$ \\
\hline SMC1207-200 & $0.5(S)$ & $0.5(\mathrm{~S})$ & $0.5(S)$ & $2(\mathrm{~S})$ & $64(\mathrm{R})$ & $128(\mathrm{R})$ & $0.125(\mathrm{~S})$ & $0.5(S)$ & $0.06(\mathrm{~S})$ & $1(\mathrm{~S})$ & $0.06(\mathrm{~S})$ & $4 / 4(S)$ & $0.25(S)$ \\
\hline \multirow[t]{2}{*}{ SMC1208-086 } & $2(S)$ & $2(S)$ & $1(\mathrm{~S})$ & $4(\mathrm{~S})$ & $64(\mathrm{R})$ & $128(\mathrm{R})$ & $0.125(\mathrm{~S})$ & $1(\mathrm{~S})$ & $0.06(\mathrm{~S})$ & $1(\mathrm{~S})$ & $0.06(\mathrm{~S})$ & 4/4 (S) & $0.5(\mathrm{~S})$ \\
\hline & \multicolumn{13}{|c|}{ TGC-IR mutants } \\
\hline SMC1204-109-IR & $>64$ (R) & $64(\mathrm{R})$ & $64(\mathrm{R})$ & $>64$ (R) & $>64$ (R) & 128 (R) & 2 (I) & $4(\mathrm{~S})$ & $0.125(\mathrm{~S})$ & $1(\mathrm{~S})$ & $0.5(\mathrm{~S})$ & 64/4 (I) & $1(\mathrm{~S})$ \\
\hline SMC1207-200-IR & $>64$ (R) & $>64(\mathrm{R})$ & $>64(\mathrm{R})$ & $>64(\mathrm{R})$ & $>64(\mathrm{R})$ & $128(\mathrm{R})$ & $4(\mathrm{R})$ & 4 (S) & $0.125(\mathrm{~S})$ & $0.5(S)$ & $0.5(S)$ & 64/4 (I) & $1(\mathrm{~S})$ \\
\hline SMC1208-086-IR & $>64$ (R) & $64(\mathrm{R})$ & $64(\mathrm{R})$ & $32(\mathrm{R})$ & $>64$ (R) & $128(\mathrm{R})$ & $1(\mathrm{~S})$ & $1(\mathrm{~S})$ & $0.06(\mathrm{~S})$ & $0.25(\mathrm{~S})$ & $0.06(\mathrm{~S})$ & $8 / 4(\mathrm{~S})$ & $0.5(\mathrm{~S})$ \\
\hline
\end{tabular}




\subsection{Biofilm Formation and Serum Resistance}

Biofilm formation decreased in three TGC-IR strains compared with that of their parental TGC-S K. pneumoniae strains, although the difference was significant only in SMC1204-109-IR $(p=0.0425)$ (Figure 3A).

A

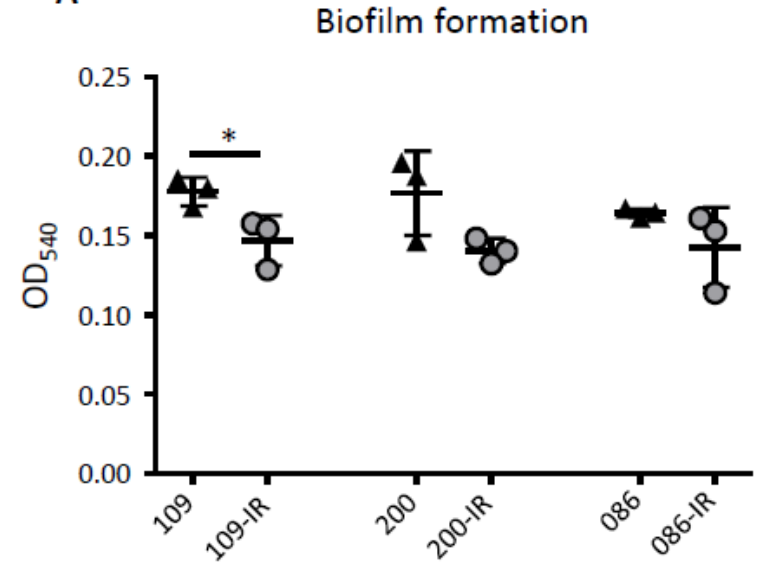

B

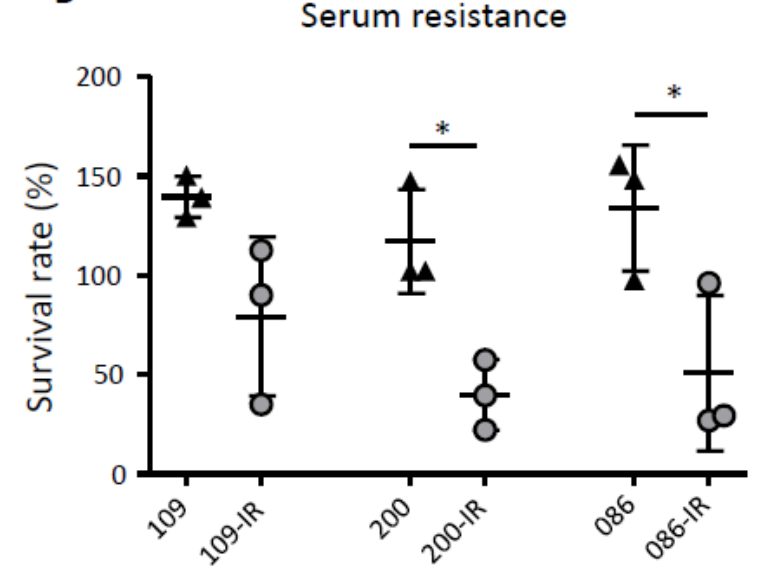

Figure 3. Results of biofilm formation and serum resistance assays. (A) Biofilm formation of TGC-S and TGC-IR K. pneumoniae strains. The biofilm was stained with crystal violet and quantified by measuring the absorbance at $540 \mathrm{~nm}$. (B) The survival rate of $K$. pneumoniae strains was determined after $3 \mathrm{~h}$ of incubation with human serum. Heat-inactivated serum (HIS) was used as a negative control. *, $p<0.05$.

The survival rates of the TGC-S strains and TGC-IR mutants were evaluated in the presence of NHS over a 3-h period. The survival rates of the three TGC-IR mutants were lower than those of their parental TGC-S strains (Figure 3B). Although the reduction in survival was not significant in one mutant (SMC1204-109-IR), it was significant in the other two ( $p=0.0131$ and 0.0462).

\subsection{Fruit Fly and Macrophage Infection}

Survival rates of D. melanogaster (fruit fly) against TGC-S and TGC-IR K. pneumoniae strains were evaluated (Figure 4A-C). While SMC1204-109-IR showed significantly decreased fly-killing ability compared with that of its parent strain $(p<0.005)$ (Figure 4A), the other TGC-IR mutants did not (Figure 4B,C). In addition, we evaluated the viable bacteria inside the fruit flies after $72 \mathrm{~h}$ of infection. The infected flies were homogenized and plated to calculate the CFU of infecting bacteria (Figure 4D). While many viable colonies $\left(1 \times 10^{3}\right.$ to $\left.5 \times 10^{5} \mathrm{CFU} / \mathrm{fly}\right)$ were identified in TGC-S strains, no viable bacteria were found when the TGC-IR mutants were administered.

The survival of the bacterial strains inside macrophages was also evaluated (Figure 5). The survival rates of three TGC-IR mutants were significantly lower than those of their parental TGC-S strains after $20 \mathrm{~h}$ of macrophage infection. 

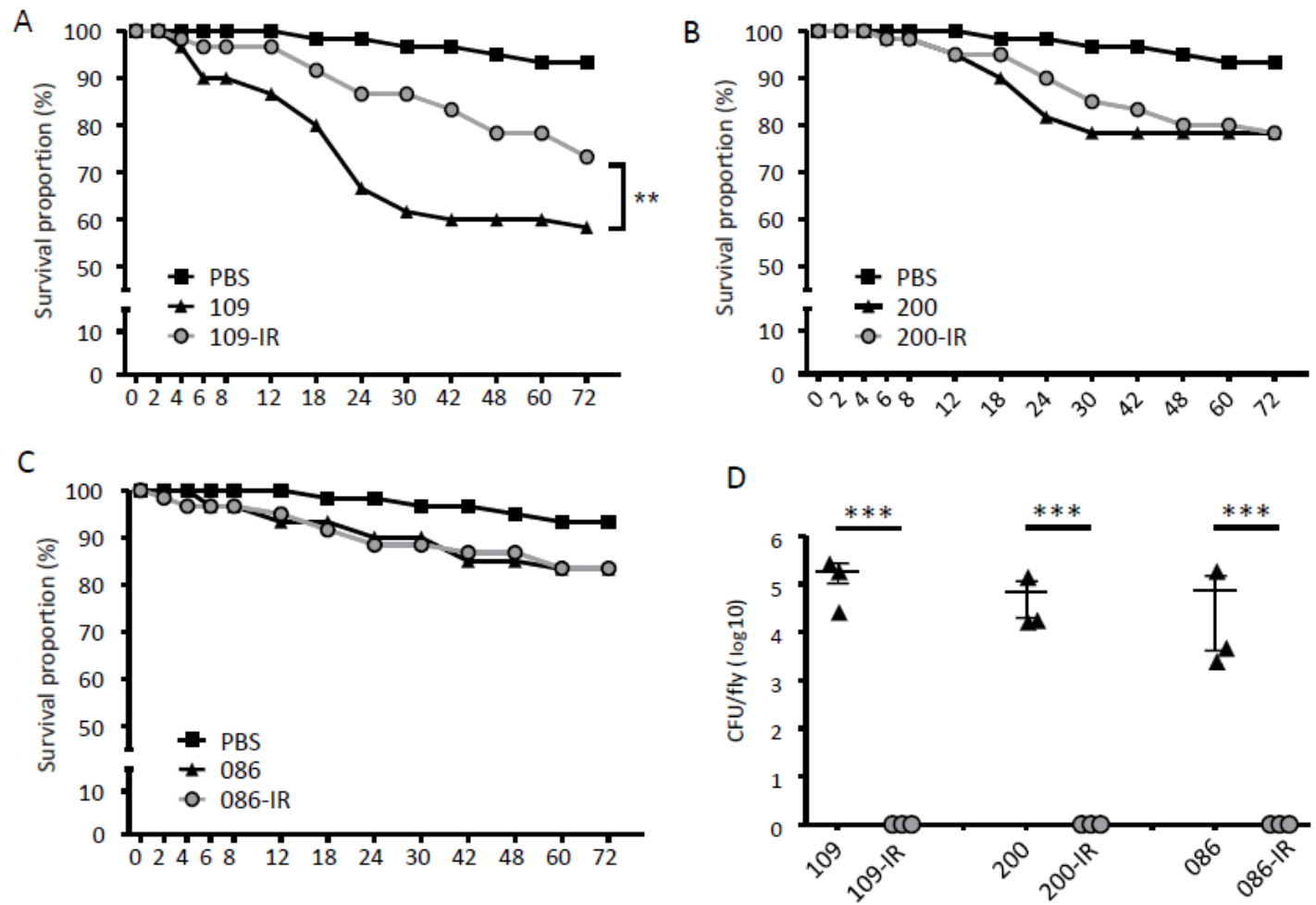

Figure 4. Results of fruit fly infections. (A-C) Survival of flies infected with TGC-S and TGC-IR strains. Fifteen flies were infected with each bacterial strain in mid-log phase $\left(\mathrm{OD}_{600}=0.5\right)$. (D) The number of surviving colonies of bacterial strains in the flies after $72 \mathrm{~h}$ of infection. The dots indicate the number of CFUs in a single fly. ${ }^{* * *}, p<0.0001$.

\section{Macrophage infection (20 h)}

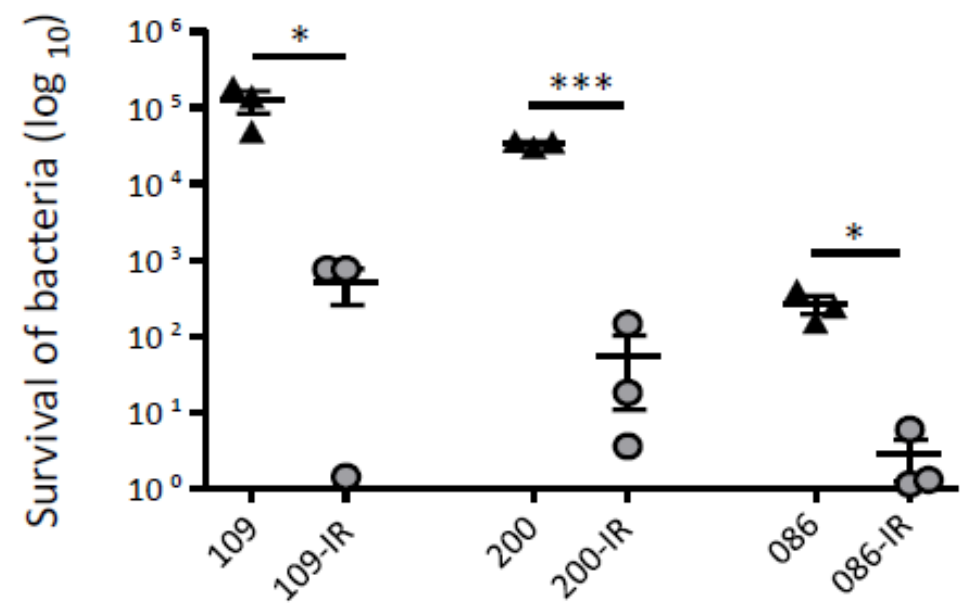

Figure 5. Result of macrophage infection assay. Survival rates of TGC-S and TGC-IR strains inside macrophages (J774A.1). The survival percentage of bacteria after $20 \mathrm{~h}$ was calculated by comparing to the CFUs at the $0 \mathrm{~h}$ time point. ${ }^{*}, p<0.05 ;{ }^{* * *}, p<0.0001$.

\subsection{Alterations in rpsJ, ramR, soxR, acrR, and marR Genes in TGC-IR Mutants}

Amino acid variations in the rpsJ gene (ribosomal S10 protein) and transcriptional regulator genes $(\operatorname{ramR}, \operatorname{sox} R, \operatorname{acr} R$, and marR) in TGC-IR mutants were investigated. Amino acid alteration in RpsJ was 
identified only in SMC1207-200-IR (Table 3) [26]. Mutations in the ramR gene were identified in all three TGC-IR mutants, but the mutations were different between them. These three mutations in the ramR gene cause a frameshift and premature stop: a deletion of four nucleotides at position 8 to 11 in SMC1204-109-IR, an insertion of adenine at position 30 in SMC1207-200-IR, and a deletion of eight nucleotides at position 128 to 135 in SMC1208-086-IR (Table 3). SMC1207-200-IR had an additional mutation in the soxR gene, an insertion of cytosine at position 94 . No alterations were identified in $a c r R$ or marR genes between TGC-S and TGC-IR strains.

Table 3. Mutations in the rpsJ gene and transcription regulator genes in TGC-IR mutants compared with that of their parental TGC-S strains.

\begin{tabular}{cccc}
\hline Genes & SMC1204-109-IR & SMC1207-200-IR & SMC1208-086-IR \\
\hline$r p s J$ & - & V57L & - \\
$\operatorname{ramR}$ & 4-bp deletion at nt 8 to 11 & A insertion at nt 30 & 8-bp deletion at nt 128 to 135 \\
$\operatorname{sox} R$ & - & C insertion at nt 94 & - \\
$\operatorname{acr} R$ & - & - & - \\
$\operatorname{marR}$ & - & - & - \\
\hline
\end{tabular}

\subsection{Expression of Genes Related to Tigecycline Resistance}

We measured the mRNA expression levels of genes that are known to be associated with tigecycline resistance in K. pneumoniae, using the qRT-PCR method. The transcriptional activator, RamA, was up-regulated significantly in all three TGC-IR mutants, compared with that of their parental TGC-S strains ( $p$-values of 0.0059, 0.0009, and 0.0014) (Figure 6A). However, the expression levels of the efflux pump genes, $a \operatorname{cr} B, \operatorname{oq} x B$, and $\operatorname{kpg} B$, were not different between TGC-S strains and TGC-IR mutants (Figure 6B).

A

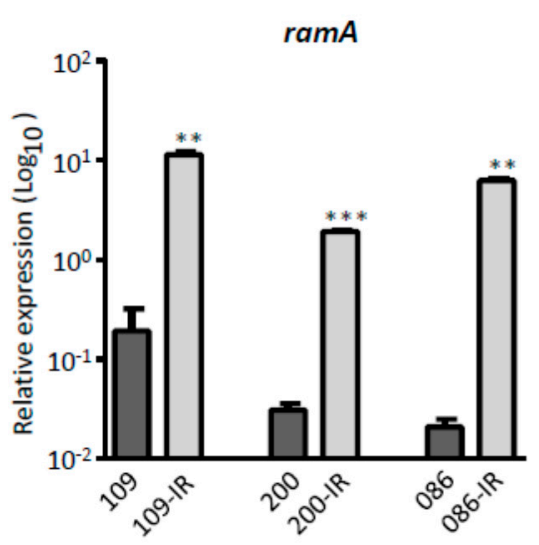

B

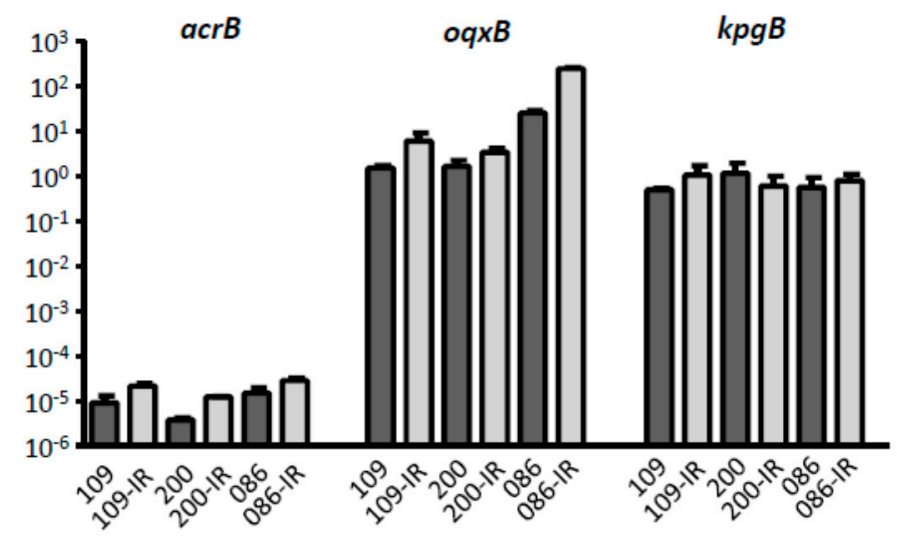

Figure 6. Expression levels of genes related to tigecycline resistance. (A) The expression level of the transcriptional activator gene $\operatorname{ram} A$. (B) The expression levels of three efflux pump genes, acrB, oqxB, and $\operatorname{kpgB}$. The fold-changes were calculated using the comparative threshold cycle $\left(\Delta \Delta \mathrm{C}_{\mathrm{T}}\right)$ method. ${ }^{* *}, p<0.001 ;{ }^{* * *}, p<0.0001$.

\section{Discussion}

In the present study, we report that the acquisition of tigecycline resistance may lead to the decreased HV phenotype, resulting in decreased virulence in K. pneumoniae clinical strains. Three tigecycline-susceptible HV K. pneumoniae isolates used in this study belonged to ST23 and showed the K1 serotype. Because hypermucoviscous K. pneumoniae are highly invasive, they have emerged as clinically important pathogens responsible for serious infections [3]. Serotype K1 is the most common among the hypermucoviscous K. pneumoniae [1], and the close association with the 
serotype and genotype ST23 is well-known $[7,27]$. Thus, the emergence of antimicrobial resistance in this pathogenic clone should be a concern [28].

Reduced hypermucoviscosity in tigecycline resistance-induced K. pneumoniae ST23 strains was identified in both the string test and mucoviscosity assay. The decreased mucoviscosity was closely associated with reduced production of CPS. Although the HV phenotype and CPS production are well-known determinants of virulence in K. pneumoniae [4], decreased virulence in tigecycline resistance-induced ST23 strains was verified, with low serum resistance and low survival rates of bacteria within fruit flies and macrophages.

Several studies have revealed that antimicrobial resistance in HV K. pneumoniae strains is relatively low compared to that of non-HV strains [5,29]. The three clinical strains included in this study were also susceptible to most antimicrobial agents except ampicillin. However, the development of tigecycline resistance leads to increased resistance to other antimicrobial agents, although the increase was lower than for tigecycline. Thus, some universal changes in the development of tigecycline resistance may affect susceptibility to other antimicrobial agents.

In a previous study, it was reported that the acquisition of colistin resistance in HV K. pneumoniae ST23 strains was accompanied by reduced capsule production, impaired virulence, and a significant fitness cost [16]. However, no change in resistance to other antimicrobial agents was observed in that study. Thus, the mechanisms responsible for decreased virulence accompanied with reduced $\mathrm{HV}$ and CPS production may be different between the processes of induction of tigecycline and colistin resistance.

Many studies have suggested that the efflux pump AcrAB contributes to tigecycline resistance in K. pneumoniae [12,30]. It was also shown that AcrAB can be up-regulated by ramR mutations and subsequently by ram $A$ activation [31]. We identified mutations in $r a m R$ and increased transcription of the $\operatorname{ram} A$ gene in tigecycline resistance-induced mutants. However, no significant increase in the expression of $a c r A B$ or other efflux pump genes was identified, and efflux pump inhibitors such as CCCP and PA $\beta N$ did not significantly decrease tigecycline MICs. Thus, efflux pumps may not contribute to tigecycline resistance and the increase in MICs for other antimicrobial agents in our K. pneumoniae mutants. Our results may indicate that $\mathrm{ram} A$ overexpression is necessarily associated with the function of efflux pumps, and that efflux pumps may not contribute to tigecycline resistance and the increase in MICs for other antimicrobial agents in our K. pneumoniae mutants. Elucidation of the key factors contributing to both antimicrobial resistance and virulence in K. pneumoniae is needed.

In summary, we found that the development of tigecycline resistance in hvKP strains may result in the decrease or loss of the HV phenotype and virulence. In addition, the induction of tigecycline resistance may be associated with an increase in resistance to other antimicrobial agents. Although development of tigecycline resistance may be due to RamA up-regulation associated with ramR mutations, efflux pumps may not be responsible for the phenomenon. Although our study has limitations that in vitro tigecycline-induced-resistant mutants were investigated and only three strains were included, the mechanisms of reduced virulence in the development of tigecycline resistance are potential targets to control hvKP infections. Thus, more investigation is required on the determinants of virulence associated with tigecycline resistance in K. pneumoniae.

Supplementary Materials: The following are available online at http:/www.mdpi.com/2076-2607/8/10/1562/s1, Table S1: Primers used in this study.

Author Contributions: Conceptualization, K.S.K.; methodology, H.L. and K.S.K.; software, D.S. and K.S.K.; validation, S.P., H.L. and K.S.K.; formal analysis, S.P. and H.L.; investigation, S.P. and H.L.; data curation, S.P., H.L. and K.S.K.; writing—original draft preparation, S.P., H.L. and K.S.K.; writing-review and editing, S.P., H.L., D.S. and K.S.K.; visualization, S.P., H.L. and K.S.K.; resources, K.S.K.; supervision, K.S.K.; project administration, K.S.K.; funding acquisition, K.S.K. All authors have contributed equally to the writing and editing of the manuscript. All authors have read and agreed to the published version of the manuscript.

Funding: This research was supported by the Basic Science Research Program through the National Research Foundation of Korea (NRF), funded by the Ministry of Science and ICT (grant NRF-2019R1A2C2004879). 
Acknowledgments: Klebsiella pneumoniae isolates used in this study were obtained from the Asian Bacterial Bank (ABB) of the Asia Pacific Foundation for Infectious Diseases (APFID) (Seoul, Korea).

Conflicts of Interest: The authors declare no conflict of interest.

\section{References}

1. Shon, A.S.; Bajwa, R.P.; Russo, T.A. Hypervirulent (hypermucoviscous) Klebsiella pneumoniae: A new and dangerous breed. Virulence 2013, 4, 107-118. [CrossRef]

2. Liu, Y.C.; Cheng, D.L.; Lin, C.L. Klebsiella pneumoniae liver abscess associated with septic endophthalmitis. Arch. Intern. Med. 1986, 146, 1913-1916. [CrossRef] [PubMed]

3. Wang, J.H.; Liu, Y.C.; Lee, S.S.J.; Yen, M.Y.; Chen, Y.S.; Wang, J.H.; Wann, S.R.; Lin, H.H. Primary liver abscess due to Klebsiella pneumoniae in Taiwan. Clin. Infect. Dis. 1998, 26, 1434-1438. [CrossRef] [PubMed]

4. Siu, L.K.; Fung, C.P.; Chang, F.Y.; Lee, N.; Yeh, K.M.; Koh, T.H.; Ip, M. Molecular typing and virulence analysis of serotype K1 Klebsiella pneumoniae strains isolated from liver abscess patients and stool samples from noninfectious subjects in Hong Kong, Singapore, and Taiwan. J. Clin. Microbiol. 2011, 49, 3761-3765. [CrossRef] [PubMed]

5. Paczosa, M.K.; Mecsas, J. Klebsiella pneumoniae: Going on the offense with a strong defense. Microbiol. Mol. Biol. Rev. 2016, 80, 629-661. [CrossRef]

6. Turton, J.F.; Englender, H.; Gabriel, S.N.; Turton, S.E.; Kaufmann, M.E.; Pitt, T.L. Genetically similar isolates of Klebsiella pneumoniae serotype K1 causing liver abscesses in three continents. J. Med. Microbiol. 2007, 56, 593-597. [CrossRef]

7. $\quad$ Chung, D.R.; Lee, S.S.; Lee, H.R.; Kim, H.B.; Choi, H.J.; Eom, J.S.; Kim, J.S.; Choi, Y.H.; Lee, J.S.; Chung, M.H.; et al. Emerging invasive liver abscess caused by K1 serotype Klebsiella pneumoniae in Korea. J. Infect. 2007, 54, 578-583. [CrossRef]

8. Pournaras, S.; Koumaki, V.; Spanakis, N.; Gennimata, V.; Tsakris, A. Current perspectives on tigecycline resistance in Enterobacteriaceae: Susceptibility testing issues and mechanisms of resistance. Int. J. Antimicrob. Agents. 2016, 48, 11-18. [CrossRef]

9. Greer, N.D. Tigecycline (Tygacil): The first in the glycylcycline class of antibiotics. Proc. (Bayl. Univ. Med. Cent.) 2006, 19, 155-161. [CrossRef]

10. Deng, M.; Zhu, M.H.; Li, J.J.; Bi, S.; Sheng, Z.K.; Hu, F.S.; Zhang, J.J.; Chen, W.; Zue, X.W.; Sheng, J.F.; et al. Molecular epidemiology and mechanisms of tigecycline resistance in clinical isolates of Acinetobacter baumannii from a Chinese university hospital. Antimicrob. Agents Chemother. 2014, 58, 297-303. [CrossRef]

11. Osei Sekyere, J.; Govinden, U.; Bester, L.A.; Essack, S.Y. Colistin and tigecycline resistance in carbapenemase-producing Gram-negative bacteria: Emerging resistance mechanisms and detection methods. J. Appl. Microbiol. 2016, 121, 601-617. [CrossRef] [PubMed]

12. Fang, H.; Fu, Y.; Chen, Q.; Ruan, Z.; Hua, X.; Zhou, H.; Yu, Y. Tigecycline susceptibility and the role of efflux pumps in tigecycline resistance in KPC-producing Klebsiella pneumoniae. PLoS ONE 2015, 10, e0119064.

13. Beabout, K.; Hammerstrom, T.G.; Perez, A.M.; Magalhães, B.F.; Prater, A.G.; Clements, T.P.; Arias, C.A.; Saxer, G.; Shamoo, Y. The ribosomal S10 protein is a general target for decreased tigecycline susceptibility. Antimicrob. Agents Chemother. 2015, 59, 5561-5566. [CrossRef] [PubMed]

14. Diancourt, L.; Passet, V.; Verhoef, J.; Grimont, P.A.; Brisse, S. Multilocus sequence typing of Klebsiella pneumoniae nosocomial isolates. J. Clin. Microbiol. 2005, 43, 4178-4182. [CrossRef] [PubMed]

15. Turton, J.F.; Baklan, H.; Siu, L.K.; Kaufmann, M.E.; Pitt, T.L. Evaluation of a multiplex PCR for detection of serotypes $\mathrm{K} 1, \mathrm{~K} 2$ and $\mathrm{K} 5$ in Klebsiella sp. and comparison of isolates within these serotypes. FEMS Microbiol. Lett. 2008, 284, 247-252. [CrossRef] [PubMed]

16. Choi, M.J.; Ko, K.S. Loss of hypermucoviscosity and increased fitness cost in colistin-resistant Klebsiella pneumoniae sequence type 23 strains. Antimicrob. Agents Chemother. 2015, 59, 6763-6773. [CrossRef] [PubMed]

17. Clinical and Laboratory Standards Institute (CLSI). Performance Standards for Antimicrobial Susceptibility Testing: Twenty-Ninth Informational Supplement M100-S29; CLSI: Wayne, NJ, USA, 2019.

18. European Committee on Antimicrobial Susceptibility Testing (EUCAST). Breakpoint Tables for Interpretation of Mics and Zone Diameters. Version 10.0. 2020. Available online: https://www.eucast.org/fileadmin/src/ media/PDFs/EUCAST_files/Breakpoint_tables/v_10.0_Breakpoint_Tables.pdf (accessed on 25 January 2020). 
19. Walker, K.A.; Miner, T.A.; Palacios, M.; Trzilova, D.; Frederick, D.R.; Broberg, C.A.; Sepulveda, V.E.; Quinn, J.D.; Miller, V.L. A Klebsiella pneumoniae regulatory mutant has reduced capsule expression but retains hypermucoviscosity. MBio 2019, 10, e00089-19. [CrossRef]

20. Pan, Y.J.; Lin, T.L.; Hsu, C.R.; Wang, J.T. Use of a Dictyostelium model for isolation of genetic loci associated with phagocytosis and virulence in Klebsiella pneumoniae. Infect. Immun. 2011, 79, 997-1006. [CrossRef]

21. Lee, H.; Shin, J.; Chung, Y.J.; Park, M.; Kang, K.J.; Baek, J.Y.; Shin, D.; Chung, D.R.; Peck, K.R.; Song, J.H.; et al. Co-introduction of plasmids harbouring the carbapenemase genes, $b l a_{\mathrm{NDM}-1}$ and $b l a_{\mathrm{OXA}-232}$, increases fitness and virulence of bacterial host. J. Biomed. Sci. 2020, 27,1-8. [CrossRef]

22. Lee, H.; Baek, J.Y.; Kim, S.Y.; Jo, H.; Kang, K.; Ko, J.H.; Cho, S.Y.; Chung, D.R.; Peck, K.R.; Song, J.H.; et al. Comparison of virulence between matt and mucoid colonies of Klebsiella pneumoniae coproducing NDM-1 and OXA-232 isolated from a single patient. J. Microbiol. 2018, 56, 665-672. [CrossRef]

23. Choi, E.; Kim, H.; Lee, H.; Nam, D.; Choi, J.; Shin, D. The iron-sensing Fur regulator controls expression timing and levels of Salmonella pathogenicity island 2 genes in the course of environmental acidification. Infect. Immun. 2014, 82, 2203-2210. [CrossRef] [PubMed]

24. Villa, L.; Feudi, C.; Fortini, D.; Brisse, S.; Passet, V.; Bonura, C.; Endimiani, A.; Mammina, C.; Ocampo, A.M.; Jimenez, J.N.; et al. Diversity, virulence, and antimicrobial resistance of the KPC-producing Klebsiella pneumoniae ST307 clone. Microb. Genom. 2017, 3, e000110. [CrossRef] [PubMed]

25. Lee, J.Y.; Chung, E.S.; Ko, K.S. Transition of colistin dependence into colistin resistance in Acinetobacter baumannii. Sci. Rep. 2017, 7, 14216. [CrossRef] [PubMed]

26. Fang, L.; Chen, Q.; Shi, K.; Li, X.; Shi, Q.; He, F.; Zhou, J.; Yu, Y.; Hua, X. Step-wise increase in tigecycline resistance in Klebsiella pneumoniae associated with mutations in ramR, lon and rpsJ. PLoS ONE 2016, 11, e0165019. [CrossRef] [PubMed]

27. Holmås, K.; Fostervold, A.; Stahlhut, S.G.; Struve, C.; Holter, J.C. Emerging K1 serotype Klebsiella pneumoniae primary liver abscess: Three cases presenting to a single university hospital in Norway. Clin. Case Rep. 2014, 2, 122-127. [CrossRef] [PubMed]

28. Lin, Y.T.; Cheng, Y.H.; Chuang, C.; Chou, S.H.; Liu, W.H.; Huang, C.H.; Yang, T.C.; Kreiswirth, B.N.; Chen, L. Molecular and clinical characterization of multidrug-resistant and hypervirulent Klebsiella pneumoniae strains from liver abscess in Taiwan. Antimicrob. Agents Chemother. 2020, 64, e00174-20. [CrossRef]

29. Lee, C.R.; Lee, J.H.; Park, K.S.; Kim, Y.B.; Jeong, B.C.; Lee, S.H. Global dissemination of carbapenemase-producing Klebsiella pneumoniae: Epidemiology, genetic context, treatment options, and detection methods. Front. Microbiol. 2016, 7, 895. [CrossRef]

30. Sheng, Z.K.; Hu, F.; Wang, W.; Guo, Q.; Chen, Z.; Xu, X.; Zhu, D.; Wang, M. Mechanisms of tigecycline resistance among Klebsiella pneumoniae clinical isolates. Antimicrob. Agents Chemother. 2014, 58, 6982-6985. [CrossRef]

31. Chiu, S.K.; Huang, L.Y.; Chen, H.; Tsai, Y.K.; Liou, C.H.; Lin, J.C.; Siu, L.K.; Chang, F.Y.; Yeh, K.M. Roles of $\operatorname{ramR}$ and tet $(A)$ mutations in conferring tigecycline resistance in carbapenem-resistant Klebsiella pneumoniae clinical isolates. Antimicrob. Agents Chemother. 2017, 61, e00391-17. [CrossRef]

(C) 2020 by the authors. Licensee MDPI, Basel, Switzerland. This article is an open access article distributed under the terms and conditions of the Creative Commons Attribution (CC BY) license (http://creativecommons.org/licenses/by/4.0/). 